\title{
Semantic cues, rhyme cues, and two varieties of recognition memory
}

\author{
DAVID L. HORTON and TIMOTHY J. PAVLICK \\ University of Maryland, College Park, Maryland
}

\begin{abstract}
The results of two experiments are reported that involved the use of both a recognition and a cued-recall task. In both experiments, the cue-target relationship was based on either meaning or rhyme. The only difference between these experiments was rate of presentation (5.0 or $1.5 \mathrm{sec}$ ) during study. The results indicate that targets were more likely to be both recognized and recalled when semantic cues appeared at study and in cued recall. Target items were more likely to be only recognized when rhyme cues appeared at study and in cued recall. The results are discussed with respect to retrieval-based recognition (recognition accompanied by recall) and familiarity-based recognition (recognition only). It is suggested that these two types of recognition are often inversely related, but they are also reflective of different memory systems.
\end{abstract}

Horton, Pavlick, and Moulin-Julian (in press) have proposed a dual-process account of recognition memory that is quite similar to earlier accounts, such as those of Mandler (1980) and Jacoby and Dallas (1981). In the Horton et al. proposal, retrieval-based recognition and familiaritybased recognition are the two processes involved in recognition. Familiarity-based recognition relies on sensoryperceptual information, and retrieval-based recognition relies on processes similar to those that influence recall. To this point, this proposal is in conceptual agreement with Mandler (1980) and Jacoby and Dallas (1981). However, Horton et al. go on to suggest that retrieval-based recognition is an episodic memory process and that increases in the quality of information in the episodic system lead to increases in both retrieval-based recognition and recall. In contrast, they propose that familiarity-based recognition operates in terms of sensory-perceptual features in semantic memory, much like those involved in pattern recognition. Increases in familiarity-based recognition occur as the quality of information in the episodic memory system declines. In other words, retrieval-based recognition and familiarity-based recognition tend to be inversely related.

The experiments reported by Horton et al. involve the use of a paradigm in which both recognition and recall of target words are measured. In this paradigm, four outcomes are possible. Targets may be remembered (i.e., recognized and recalled), recognized only, recalled only, or forgotten (i.e., not recognized and not recalled).

The results of two experiments reported by Horton et al. show that increases in the associative relationship between cue and target members of word pairs presented at study are directly related to performance in the remembered outcome but are inversely related to performance in the

Computer services were provided by the Computer Science Center at the University of Maryland. Requests for reprints should be sent to D. L. Horton, Department of Psychology, University of Maryland, College Park, MD 20742. recognized-only outcome. Performance in the remembered outcome is seen as reflecting retrieval-based recognition, and performance in the recognized-only outcome reflects familiarity-based recognition. Combining these two indices to obtain overall recognition performance results in a composite index that is not significantly related to the index of associative relationship.

The results of a third experiment reported by Horton et al. indicate that when processing instructions emphasize semantic aspects of the materials, such as pleasantness ratings or category membership, memory performance is best in the remembered outcome. However, when processing instructions emphasize nonsemantic aspects of the materials, such as rhyme rating or common first letter, memory performance is best in the recognizedonly outcome. When these two outcomes are combined as an index of overall recognition performance, variations in relational processing (category membership vs. common first letter) have no effect and the effects of variations in individual item processing (pleasantness rating vs. rhyme rating) are reduced. Horton et al. State that it is not plausible to assert that factors such as associative relatedness or semantic-nonsemantic processing, which have a substantial effect on recall, have no effect on recognition unless recognition involves two processes that tend to be inversely related and are never complementary.

The two experiments reported here were designed to further investigate these two varieties of recognition in a context in which type of processing was manipulated in terms of cue-target relationships of a semantic or nonsemantic variety. Type of processing was a betweensubject variable, and the cue-target relationship was either meaning-related or rhyme-related. The design of the two experiments was identical, except for presentation rate $(5.0$ or $1.5 \mathrm{sec})$. With respect to the comparison of the remembered and recognized-only outcomes, it was expected that performance would be best in the remembered outcome with semantic cues and best in the recognizedonly outcome with rhyme cues. However, there should 
be no main effect of cue type. In addition, it was expected that performance would be substantially depressed in the remembered outcome for the faster presentation rate, relative to the slower presentation rate. This prediction was based, in part, on a study reported by Nelson and McEvoy (1979) in which it was found there was an advantage for nonsemantic cues in cued recall at a fast presentation rate $(1.5 \mathrm{sec})$ but an advantage for semantic cues at a slower rate $(3.0 \mathrm{sec})$.

\section{EXPERIMENT 1}

\begin{abstract}
Method
Materials. The semantic cue-target pairs were selected from controlled association norms collected by Douglas L. Nelson and his associates (Nelson, personal communication, November 1983). The semantically related pairs consisted of 30 weakly related pairs (associative strength less than .08) that had been employed in a prior study (Diehl \& Horton, 1988). Each of the 30 target words was paired with a word that rhymed with it. For each pair type list, an additional eight pairsfour at the beginning and four at the end-were included to control for primacy and recency effects. The words in these pairs did not appear in the retention tests. For the semantic pairs, the list could be divided into three sets of 10 words, which varied in common set size. This factor refers to the number of extra list words (one, two, or three) associatively linked to both the cue and the target members of a pair according to Nelson's norms. This factor does not apply to to the rhyme pair list.

The recognition task consisted of 90 words: 30 target words common to both lists, 30 semantic words (each of which was semantically related to one of the target words), and 30 rhyme words (each of which rhymed with one of the target words).

Subjects. Forty undergraduate introductory psychology students participated in Experiment 1. The students received extra course credit for their participation. Twenty subjects were randomly assigned to each cuetype condition. All participants gave informed consent by signing an agreement, and they were treated in accordance with the ethical principles of the American Psychological Association.

Procedure. The subjects were run in small groups (1-6). A carousel projector was used to present a series of 38 word pairs for $5 \mathrm{sec}$ each. The subjects were instructed to remember the capitalized word in each pair (i.e., target) and to look for a relationship between the two words in order to help them remember the target word. A 10-min numerical problem-solving distractor task immediately followed pair presentation.

Following the problem-solving task, the subjects were asked to complete the recognition task. The subjects were instructed to identify each word as old (i.e., target) or new (i.e., a distractor). The distractors were identical for both cue-type conditions. After this, the cued-recall test was given. It consisted of a list of the 30 cue words presented during study, and the subjects were asked to write next to each cue word the word it was paired with during study.
\end{abstract}

\section{Results and Discussion}

An analysis of variance (ANOVA) was performed on both false alarms during recognition and intrusions during recall as a check on the set induced during encoding in each condition. As expected, rhyme false alarms were more common than semantic false alarms when rhyme cues occurred during encoding (9.00 vs. 6.65$)$, and the opposite was the case when semantic cues occurred during encoding (6.70 vs. 5.80$)$. The interaction of cue type and type of false alarm yielded $F(1,38)=10.18, p<$ .01 . The same pattern was found for intrusions, with rhyme cues leading to more rhyme intrusions (13.25 vs. 4.60 ) and semantic cues leading to more semantic intru- sions (13.60 vs. 1.05). The interaction in this case yielded $F(1,38)=118.15, p<.0001$.

The comparison of primary interest in this study involved the remembered and recognized-only outcomes. For clarity of presentation, the results are reported as proportions. The results for these outcomes are shown in Table 1 . As can be seen, the expected interaction between cue type and memory outcome is clear-cut. This is supported by an ANOVA that showed a significant interaction effect $[F(1,38)=9.20, p<.005]$. There was also a significant interaction between memory outcome and common set size $[F(2,76)=3.13, p<.05]$. This interaction arises because, with semantic cues, performance in the remembered outcome was systematic and was best with the small (one) common set size, and performance in the recognized-only outcome was also systematic but best with the largest (three) common set size. This trend tended to occur with rhyme cues, for no apparent reason, but it was much less systematic, resulting in a significant interaction.

The findings from Experiment 1 clearly support the distinction between retrieval-based recognition (the remembered outcome) and familiarity-based recognition (the recognized-only outcome). An inverse relationship obtains between outcomes as a function of cue type and, as expected from Diehl and Horton's (1988) findings, for semantic cues an inverse relationship is found between outcomes as a function of common set size.

An ANOVA was also performed on the recalled-only and forgotten outcomes, with the theoretically uninteresting result that the forgotten outcome occurred more than twice as often as the recalled-only outcome $[F(1,38)=$ $30.10, p<.0001]$.

\section{EXPERIMENT 2}

As noted previously, Experiment 2 was identical to Experiment 1 , except that the presentation rate was $1.5 \mathrm{sec}$ per pair rather than $5.0 \mathrm{sec}$ per pair. The principle effect of this faster presentation rate was expected to be a major reduction in performance in the remembered outcome, relative to that observed in Experiment 1, due to a reduced opportunity for semantic processing.

\section{Method}

Forty-four undergraduate introductory psychology students participated in Experiment 2. The students received extra course credit for their participation. Twenty-two subjects were randomly assigned to each cue-type condition. All participants gave informed consent by signing an agreement, and they were treated in accordance with the ethical principles of the American Psychological Association.

Table 1

Proportion of Outcomes in the Remembered and Recognized-Only Outcomes as a Function of Cue Type

\begin{tabular}{lcc}
\hline & \multicolumn{2}{c}{ Cue } \\
\cline { 2 - 3 } Outcome & Semantic & Rhyme \\
\hline Remembered & .45 & .31 \\
Recognized-Only & .30 & .43 \\
\hline
\end{tabular}


Table 2

Proportion of Outcomes in the Remembered and Recognized-Only Outcomes as a Function of Cue Type

\begin{tabular}{lcc}
\hline & \multicolumn{2}{c}{ Cue } \\
\cline { 2 - 3 } Outcome & Semantic & Rhyme \\
\hline Remembered & .28 & .21 \\
Recognized-Only & .34 & .44 \\
\hline
\end{tabular}

\section{Results and Discussion}

In contrast to the false-alarm pattern found in Experiment 1 , false alarms were generally more common, particularly when rhyme cues were presented at encoding, and rhyme-related distractors appeared as false alarms more often than did semantic-related distractors in both cue-type conditions. While both of these effects are consistent with the expectation of reduced semantic processing at the faster presentation rate, they are only marginally significant $(p<.06)$.

In the case of intrusions, the pattern observed in Experiment 2 is comparable to that observed in Experiment 1 . When rhyme cues appeared at study, rhyme intrusions were more common than semantic intrusions (15.86 vs. 5.19); when semantic cues appeared at study, the opposite pattern of intrusions (16.91 vs. 2.52) was observed. This is supported by a significant interaction between cue type and type of intrusion $[F(1,42)=$ $148.45, p<.0001]$. This relationship is to be expected even with reduced semantic processing, since the general nature of the cue-target relationship should be obvious to subjects in both conditions.

The results of primary interest in this study are those comparing the remembered and recognized-only outcomes. For clarity of presentation, the results are reported as proportions. Those results are given in Table 2 .

As in Experiment 1, there was a significant interaction between cue type and memory outcome, with semantic cues leading to better performance in the remembered outcome and rhyme cues leading to superior performance in the recognized-only outcome $[F(1,42)=7.86, p<.01]$. However, in contrast to Experiment 1 but in accord with expectations, there was a significant effect of memory outcome, with much better performance in the recognizedonly outcome $[F(1,42)=26.12, p<.0001]$.

The only other effect to reach significance was the interaction between memory outcome, cue type, and common set size $[F(2,84)=4.59, p<.02]$. The interaction arises because, with semantic cues, performance in the remembered outcome was systematic and best with the small (one) common set size, and performance in the recognized-only outcome was systematic and best with the large (three) common set size. With rhyme cues, such a trend was not observed, and there is no reason why it should have been observed since the common-set-size factor was only defined for semantic relatedness. However, the observed inverse relationship between the remembered and recognizedonly outcomes across levels of the common-set-size factor for semantic cues is of some theoretical significance for our proposal.

As was the case for Experiment 1, the analysis of the recalled-only and forgotten outcomes showed that the forgotten outcome occurred twice as often as the recalledonly outcome $[F(1,42)=111.31, p<.001]$.

\section{GENERAL DISCUSSION}

The results of the experiments reported here are quite consistent with the proposal introduced by Horton et al. (in press). In both experiments, performance in the remembered outcome was inversely related to performance in the recognized-only outcome. This held across cue type and, for the semantic cue condition, it held across levels of common set size. These observations clearly support the distinction between retrieval-based recognition and familiarity-based recognition as qualitatively different processes that are often inversely related. We do not propose that these recognition processes always lead to inversely related results but, since they are seen as being oppositional, they should not produce complementary findings.

The results reported here are also consistent with the findings reported by Horton et al. concerning the integration of type of recognition process and the levels of processing manipulation. Although statistical comparisons between the two experiments reported here were not carried out, the results are clearly in line with those of Nelson and McEvoy (1979) concerning the relationship between levels of processing and rate of presentation. A comparison of Tables 1 and 2 reveals a distinct suppression of performance in the remembered outcome with the faster presentation rate. In contrast, performance in the recognized-only outcome was about the same for both presentation rates. In addition, the falsealarm and intrusion findings are consistent with expectations.

In conclusion, it seems quite clear that the overall pattern of findings reported here is very consistent with the proposal for retrieval-based and familiarity-based recognition as qualitatively different processes. In addition, the reported results are consistent with prior findings concerning levels of processing and presentation rate manipulations.

\section{REFERENCES}

DieHL, V. A., \& HoRTon, D. L. (1988). Encoding context effects in recognition and cued recall. Bulletin of the Psychonomic Society, 26, 393-394.

Horton, D. L., Pavlick, T. J., \& Moulin-Julian, M. W. (in press). Retrieval-based and familiarity-based recognition and the quality of information in episodic memory. Journal of Memory \& Language.

JACOBY, L. L., \& DALLAS, M. (1981). On the relationship between autobiographical memory and perceptual learning. Journal of Experimental Psychology: General, 110, 306-340.

MANDLER, G. (1980). Recognizing: The judgment of previous occurrence. Psychological Review, 87, 252-271.

Nelson, D. L., \& McEvoy, C. L. (1979). Encoding context and set size. Journal of Experimental Psychology: Human Learning \& Memory, 5, 292-314.

(Manuscript received July 3, 1992). 